\title{
Weight Loss Approach during Routine Follow-up is Effective for Obstructive Sleep Apnea Hypopnea Syndrome Subjects Receiving Nasal Continuous Positive Airway Pressure Treatment
}

\author{
Hiroko FUJII' ${ }^{1 *}$, Masayuki MIYAMOTO², Tomoyuki MIYAMOTO² and Takashi MUTO1 \\ ${ }^{1}$ Department of Public Health, Dokkyo Medical University School of Medicine, 880 Kitakobayashi, Mibu, \\ Shimotsuga, Tochigi 321-0293, Japan \\ ${ }^{2}$ Department of Neurology, Center of Sleep Medicine, Dokkyo Medical University School of Medicine, 880 \\ Kitakobayashi, Mibu, Shimotsuga, Tochigi 321-0293, Japan
}

Received March 10, 2009 and accepted November 26, 2009

\begin{abstract}
The present study investigated the effectiveness of a weight loss program during routine medical follow-up with regularity on promoting weight reduction in obese obstructive sleep apnea hypopnea syndrome (OSAHS) subjects receiving continuous positive airway pressure treatment (CPAP). A total of 10 male obese OSAHS subjects treated with CPAP were enrolled in the present study that was an intervention study without a control and had a pre-post test study design. The age was 50.7 (7.8) (mean (SD)) years, and body mass index was 30.7 (2.5) $\mathrm{kg} / \mathrm{m}^{2}$. A 4-month weight loss program was developed, using a combined approach of diet and physical activity based on individual counseling with behavioral approach. At 4 months, weight was significantly decreased compared with the baseline value $(88.4(8.9) \mathrm{kg}$ to $86.9(8.8)$ $\mathrm{kg}, p=0.005$ ), and the mean weight loss was a $1.7 \%$ decrease from the baseline. There was significantly higher percent weight loss in the group with a CPAP duration $<30$ months, than in the group $\geq 30$ months $(2.7(1.6) \%$ vs. $0.6(0.5) \%, p=0.032)$. The present study shows that a weight loss program may be useful in reducing weight for male obese OSAHS subjects treated with CPAP.
\end{abstract}

Key words: Obstructive sleep apnea hypopnea syndrome, Continuous positive airway pressure treatment, Weight loss, Obesity

\section{Introduction}

Obesity is well recognized as a major risk factor for chronic diseases such as hypertension, hyperlipidemia, type 2 diabetes, and coronary heart disease, and it is also known as one of the major risk factors for obstructive sleep apnea hypopnea syndrome (OSAHS) ${ }^{1)}$. Daytime sleepiness by sleep disordered breathing caused by OSAHS has been a serious problem in worksites. That increases the risk of traffic accident ${ }^{2}$, occupational accidents $^{3)}$, the amount of sick leaves from the work $^{4)}$

*To whom correspondence should be addressed.

E-mail: hirofuji@dokkyomed.ac.jp and decrease of work productivity ${ }^{5}$.

Nasal continuous positive airway pressure treatment (CPAP) is the most effective standard treatment for symptoms of OSAHS. Weight loss has been shown having a positive relationship with improvement in sleep parameters in literatures ${ }^{6-8)}$. Furthermore, the need for CPAP was diminished by remarkable weight loss ${ }^{6}$. Weight loss provides not only improvement of OSAHS but also additional health benefits such as improvement of blood pressure, lipid levels and glucose metabolism, and therefore should be routinely recommended to most obese OSAHS subjects $\left.{ }^{9}, 10\right)$.

Published data on the association between CPAP and weight loss are inconsistent ${ }^{1-13)}$. Medical staff should 
stress weight loss support for obese OSAHS subject treated with CPAP, together with support for increasing CPAP adherence ${ }^{12)}$. However, the weight loss approach needs to have feasibility and efficacy in order to prevail. To our knowledge, the feasibility and efficacy of a weight loss program using behavioral approach during routine medical follow-up for obese workers who have OSAHS treated with CPAP has not been studied previously. The present study investigated whether a weight loss program lasting for 4 months could have an effect on weight reduction. Furthermore, baseline factors that might have influenced weight loss in obese OSAHS subjects with CPAP were evaluated.

\section{Subjects and Methods}

\section{Study design and participants}

The present study was an intervention study without a control and had a pre-post test study design. Participants were recruited from subjects receiving CPAP for OSAHS at Center of Sleep Medicine, Dokkyo Medical University Hospital between January and May 2008. They were diagnosed as having OSAHS based on a polysomnography in-laboratory, according to the second edition of the International Classification of Sleep Disorders ${ }^{14)}$. Inclusion criteria were as follows, (a) body mass index (BMI) $\geq 25 \mathrm{~kg} / \mathrm{m}^{2}$, (b) age at baseline $\geq 20 \mathrm{yr}$, (c) positive intention to follow the weight loss program. Exclusion criteria were the presence of untreated severe illness, use of hypnotics and drugs known to affect weight.

A total of 10 subjects participated in the weight loss program. The study protocol was approved from the ethics committee at Dokkyo Medical University. We obtained written informed consent from each subject following an explanation of the positive relationship between weight reduction and diminishing CPAP.

\section{Interventions}

The weight loss program was a combined approach of diet and physical activity constructed by individual counseling with behavioral approach. A registered dietitian met participants for a monthly counseling session lasting approximately $30 \mathrm{~min}$. We used only the intervention method of individual counseling not including group sessions, because we considered that we needed to fit our intervention schedule to each subject' schedule of consulting their doctor in order to facilitate participation in the weight loss program.

The aim of the weight loss program was to help subjects maintain preferable lifestyle behaviors that were modified during the program, after the program ended. A dietician worked with the subjects to help them rec- ognize problem lifestyle behaviors related to obesity.

The counseling included giving oral and written information in terms of weight loss, nutrition, and physical activity. The importance of dietary control and physical activity increase to achieve weight loss were shown to all subjects with common information using the manual (Niheisya Corp., Osaka, Japan) ${ }^{15}$ ). Other health information such as information on the energy content of foods at restaurants, alcoholic drinks and confectionery, and information on recipes for a healthy diet were distributed based on each subject's weight loss needs. A pedometer was distributed to each subject for objective assessment of physical activity. Subjects were encouraged at the counseling to select their own way to change their lifestyle, specifically to set the tangible goals, and to self-monitor the target behaviors and daily weight as frequently as they could. The subjects set various tangible goals, e.g. 10,000 or more number of walking steps per day, not eating snacks, limiting the amount of alcoholic drinks, etc. In addition, they changed or added lifestyle modification goals that they thought they could achieve.

\section{Measurements}

Height at baseline and weight at every monthly visit were measured wearing light clothing and no shoes. BMI was calculated as weight $(\mathrm{kg})$ divided by height squared $\left(\mathrm{m}^{2}\right)$. Weight loss was calculated as: $\mathrm{W}_{0}-\mathrm{W}_{4}$, where $\mathrm{W}_{0}$ was the weight at baseline, and $\mathrm{W}_{4}$ was the weight at 4 months, and the percent weight loss was calculated as: $\left(\mathrm{W}_{0}-\mathrm{W}_{4}\right) / \mathrm{W}_{0} \times 100$. Daily monitoring of CPAP usage was recorded by the internal memory of the CPAP devices. CPAP adherence was defined as the proportion of nights showing use for a minimum of 4 hours a night during the previous 1 month, and adherent of CPAP was defined as a subject showing CPAP adherence $\geq 70 \%$ completed $^{12,16)}$.

The participants completed questionnaires by themselves regarding sleep related measures, weight history, and lifestyle at baseline and 4 months. Sleep quality was assessed by the Pittsburgh Sleep Quality Index (PSQI) ${ }^{17)}$ Japanese version ${ }^{18)}$ and daytime sleepiness by the Epworth Sleepiness Scale (ESS) ${ }^{19)}$ Japanese version $^{20)}$. The PSQI is a 19 -item questionnaire that produces a global sleep quality score that ranges from 0 to 21 , derived from 7 component (sleep quality, sleep latency, sleep duration, sleep efficiency, sleep disturbance, hypnotics medication use and daytime dysfunction $)^{17)}$. The ESS is an 8-item questionnaire in which the subject rates the likelihood of dozing during typical daytime activities on a scale of $0-3^{19}$ ).

Stage of change with regard to healthy lifestyle behaviors is defined in 5 stages as follows: "precontem- 
plation", subjects do not have healthy lifestyle behaviors and do not plan to change their lifestyle behaviors; "contemplation", though subjects do not have healthy lifestyle behaviors now, they are thinking about changing their lifestyle behaviors and they will begin to do that in the next 6 months; "preparation", though subjects do not have healthy lifestyle behaviors now, they will begin to do that in the next 1 month; "action", subjects have healthy lifestyle behaviors now and they made the change in the past 6 months; "maintenance", subjects have healthy lifestyle behaviors now and they made the change more than 6 months ago ${ }^{21)}$. The stage of change among participants in this study was assessed by asking what preferable dietary behaviors or physical activities they were currently trying to follow in order to lose or maintain their weight. We categorized their responses into 2 categories; "precontemplation or contemplation or preparation" stage or "action or maintenance" stage. Smoking status was indicated using three categories: current smoker, former-smoker and never-smoker. Drinking status was confirmed by frequency of drinking and quantity of alcohol consumption. Habitual drinker was defined as one who takes more than approximately $20 \mathrm{~g}$ of alcohol per day for $3 \mathrm{~d}$ or more per week. Job type and occupational classification (Statistic Bureau, Ministry of Internal Affairs and Communications, Japan) was confirmed by interviewing the participants in the individual counselling. Medication proscribed by the doctor was confirmed by medical record and asking each subject.

\section{Statistical analysis}

Changes between baseline and 4 months were examined by Wilcoxon's signed-rank test for continuous variables and ordinal variables, and by McNemar's test for categorical variables. The association between the percent weight loss and CPAP duration, age, BMI, CPAP adherence, ESS, PSQI and stage of change at baseline were investigated by Spearman's rank correlation coefficient. The subjects were divided into two groups by CPAP duration greater than or less than 30 months, which was the median of all participants. Differences between two groups were compared for percent weight loss for 4 months, age and BMI at baseline, CPAP adherence at baseline and 4 months at baseline by Mann-Whitney U test. Differences between two groups were compared for prevalence of adherent of CPAP, action or maintenance stage of change at baseline and 4 months by Fisher's exact test.

In all tests, values of $p<0.05$ of two-tailed test were considered significant. All analyses were performed using SPSS 15.0J for Windows (SPSS Japan Inc., Tokyo, Japan).

\section{Results}

Table 1 shows the characteristics of the participants at baseline and 4 months. At 4 months, weight and BMI were significantly decreased compared with baseline ( $p=0.005$ and $p=0.005$, respectively). The proportion of subject with stage of change on action or maintenance of exercise behavior at 4 months increased significantly $(p=0.008)$. There were no significant differences in other factors between baseline and 4 months. The occupations of the participants were as follows: specialist and technical workers (4), clerical workers (3), administrative and managerial worker (1), sales worker (1), and production process and related worker (1).

Shorter CPAP duration was correlated with a higher degree of percent weight loss (Spearman $\mathrm{r}=0.705$, $p=0.023$ ). There was no significant correlation between the percent weight loss and other factors at baseline. There was a significantly higher percent weight loss in the group with a CPAP duration $<30$ months, than in the group $\geq 30$ months $(p=0.032)$ (Table 2$)$. There was no significant difference between the two groups with regard to CPAP adherence and other factors at baseline and 4 months.

\section{Discussion}

The present study investigated the effectiveness of a 4-month weight loss program for obese OSAHS subjects being treated with CPAP under routine medical followup. The program consisted of a combined approach to diet and physical activity with individual counseling using a behavioural approach, and it led to significant weight reduction. Furthermore, there was significant association between CPAP duration and achievement of weight loss by the study participants. Our findings indicate that a 4-month weight loss program using a self-selected behavioral approach was effective and that the timing of starting weight loss approach was important for male obese OSAHS subjects treated with CPAP, namely these OSAHS subjects should be offered a weight loss program as soon as possible, at the latest when beginning CPAP.

Our original weight loss program for 4 months encouraged the subjects to select their own way of changing their lifestyle during routine medical followup. During monthly individual counseling sessions in this program, a dietitian provided support messages and information aimed at weight reduction focusing on subjects' self-management regarding lifestyle modification. The program did not require a very low calorie diet. The program was a relatively light intervention for weight loss in a real clinical setting. In the present 
Table 1. Characteristics of participants in the weight loss program at baseline and 4 months

\begin{tabular}{|c|c|c|c|}
\hline & Baseline & 4 months & $p$-value ${ }^{\mathrm{a}}$ \\
\hline Number of patients, $n$, (Men/Women) & $10(10 / 0)$ & - & - \\
\hline Age, yr, mean (SD) & $50.7(7.8)$ & - & - \\
\hline Total number of counseling sessions for the program, time, mean (SD) & $5.2(1.0)$ & - & - \\
\hline Medication for hypertension, $\mathrm{n}(\%)$ & $2(20)$ & $2(20)$ & - \\
\hline Medication for hyperlipidemia, $\mathrm{n}(\%)$ & $1(10)$ & $1(10)$ & - \\
\hline Medication for diabetes, $\mathrm{n}(\%)$ & $0(0)$ & $0(0)$ & - \\
\hline Shift worker, n (\%) & $0(0)$ & $0(0)$ & - \\
\hline Current smoker, n (\%) & $3(30)$ & $3(30)$ & 1.000 \\
\hline Habitual drinker, n (\%) & $2(20)$ & $2(20)$ & 1.000 \\
\hline AHI at OSAHS diagnosis, time/hour, mean (SD) & $59.0(20.9)$ & - & - \\
\hline Duration from OSAHS diagnosis to baseline, month, mean (SD) & $32.5(13.8)$ & - & - \\
\hline CPAP duration, month, mean (SD) & $31.0(13.9)$ & - & - \\
\hline CPAP adherence, $\%$, mean (SD) & $63.1(24.5)$ & $66.6(33.7)$ & $0.374^{\mathrm{b}}$ \\
\hline Adherent of CPAP, n (\%) & $5(50)$ & $5(50)$ & 1.000 \\
\hline ESS, point, mean (SD) & $9.1(4.0)$ & $9.2(4.4)$ & $0.888^{\mathrm{b}}$ \\
\hline PSQI, point, mean (SD) & $3.6(2.0)$ & $3.7(2.4)$ & $0.773^{b}$ \\
\hline Weight, kg, mean (SD) & $88.4(8.9)$ & $86.9(8.8)$ & $0.005^{\mathrm{b}}$ \\
\hline Weight loss, kg, mean (SD) & - & $1.5(1.4)$ & - \\
\hline \%weight loss, \%, mean (SD) & - & $1.7(1.6)$ & - \\
\hline Body mass index, kg/m², mean (SD) & $30.7(2.5)$ & $30.3(2.4)$ & $0.005^{\mathrm{b}}$ \\
\hline Weight $1 \mathrm{yr}$ prior to baseline, $\mathrm{kg}$, mean (SD) & $86.6(8.8)$ & - & $0.072^{\mathrm{b}, \mathrm{c}}$ \\
\hline Weight at $20 \mathrm{yr}$ old, $\mathrm{kg}$, mean (SD) & $68.6(9.4)$ & - & $0.005^{\mathrm{b}, \mathrm{d}}$ \\
\hline \multicolumn{4}{|l|}{ Stage of change } \\
\hline Dietary behavior (action or maintenance), n (\%) & $4(40)$ & $7(70)$ & 0.375 \\
\hline Exercise behavior (action or maintenance), $\mathrm{n}(\%)$ & $1(10)$ & $9(90)$ & 0.008 \\
\hline
\end{tabular}

Abbreviation: AHI, Apnea-hypopnea index; OSAHS, Obstructive sleep apnea hypopnea syndrome; CPAP, nasal continuous positive airway pressure treatment; ESS, Epworth sleepiness scale; PSQI, Pittsburgh quality of sleep index.

a $p$ value was determined by McNemar's test, unless otherwise specified.

b $p$ value was determined by Wilcoxon's signed-rank test.

${ }^{c} p$ value was determined between weight $1 \mathrm{yr}$ prior to baseline and weight at baseline.

${ }^{\mathrm{d}} p$ value was determined between weight at $20 \mathrm{yr}$ old and weight at baseline.

study, $1.5 \mathrm{~kg}(1.7 \%)$ of mean weight loss over 4 months was smaller than the loss in these reports on lifestyle intervention $^{7,8)}$, and also smaller than $5-10 \%$ weight loss for significant clinical health benefit ${ }^{22}$. However, health counseling and behavioral interventions for weight reduction promoted 3-5 $\mathrm{kg}$ weight loss over $1 \mathrm{yr}$ was shown in systematic reviews of randomized control trials ${ }^{9)}$. Considering the frequency and the study period in our study, it may be safe to interpret the effect on weight loss as reasonable. In addition, it is vital for subjects to halt the trend toward weight gain during the study periods. Unfortunately, we could not confirm significant subjective improvement related to treatment. This may be due to the achievement of modest weight reduction. To confirm the long-term effect of the present findings and clarify improvement of subjective factors caused by modest weight loss, further study is warranted. In addition, perceiving improvement in subjective symptoms following modest weight loss is important for encouraging their effort in weight reduc- tion and maintenance.

The present study also showed a possibility that the earlier weight loss approach after prescribing CPAP, the greater the degree of weight reduction. Obese subjects with a shorter CPAP duration may have greater motivation for weight loss because they may not yet be adequately accustomed to CPAP, and have a stronger desire to get rid of CPAP than those with a longer duration of CPAP. A positive association between CPAP adherence at baseline and weight reduction was not confirmed in this study. This finding suggested that CPAP adherence and adherence to weight loss program might have been inconsistent among obese OSAHS subjects treated with CPAP. It was similar to the literature showing that BMI did not significantly decrease in the CPAP adherent group and the control group including those without CPAP and those who did not adhere to CPAP among middle-aged men and women ${ }^{12)}$. We assume that subjects with high CPAP adherence do not always achieve weight reduction. These may give support to the 
Table 2. Comparison of percent weight loss, CPAP adherence and lifestyle behavior stage of change with CPAP duration under 30 months or over 30 months

\begin{tabular}{|c|c|c|c|}
\hline & \multicolumn{2}{|c|}{$\mathrm{CPAP}$ duration at baseline } & \multirow{2}{*}{$p$-value ${ }^{\mathrm{a}}$} \\
\hline & $<30$ months & $\geq 30$ months & \\
\hline Number of patients, $\mathrm{n}$ & 5 & 5 & - \\
\hline Percent weight loss for 4 months, $\%$, mean (SD) & $2.7(1.6)$ & $0.6(0.5)$ & 0.032 \\
\hline \multicolumn{4}{|l|}{ Baseline factors } \\
\hline Age, yr, mean (SD) & $47.6(9.6)$ & $53.8(4.7)$ & 0.310 \\
\hline Body mass index, $\mathrm{kg} / \mathrm{m}^{2}$, mean (SD) & $30.5(2.6)$ & $31.1(2.6)$ & 0.690 \\
\hline CPAP adherence, $\%$, mean $(\mathrm{SD})$ & $64.3(6.9)$ & $62.0(36.0)$ & 0.548 \\
\hline Adherent of CPAP, $\mathrm{n}(\%)$ & $2(40.0)$ & $3(60.0)$ & $1.000^{\mathrm{b}}$ \\
\hline Dietary behavior stage of change (action or maintenance), $\mathrm{n}(\%)$ & $3(60.0)$ & $1(20.0)$ & $0.524^{\mathrm{b}}$ \\
\hline Exercise behavior stage of change (action or maintenance), $\mathrm{n}(\%)$ & $0(0)$ & $1(20.0)$ & $1.000^{\mathrm{b}}$ \\
\hline \multicolumn{4}{|l|}{ Factors at 4 months } \\
\hline CPAP adherence, $\%$, mean (SD) & $59.9(25.6)$ & $73.3(42.4)$ & 0.222 \\
\hline Adherent of CPAP, n (\%) & $1(20.0)$ & $4(80.0)$ & $0.206^{\mathrm{b}}$ \\
\hline Dietary behavior stage of change (action or maintenance), $\mathrm{n}(\%)$ & $3(60.0)$ & $4(80.0)$ & $1.000^{\mathrm{b}}$ \\
\hline Exercise behavior stage of change (action or maintenance), $\mathrm{n}(\%)$ & $5(100.0)$ & $4(80.0)$ & $1.000^{\mathrm{b}}$ \\
\hline
\end{tabular}

Abbreviation: CPAP, nasal continuous positive airway pressure treatment.

${ }^{a} p$ value was determined by Mann-Whitney U test, unless otherwise specified.

${ }^{\mathrm{b}} p$ value was determined by Fisher's exact test.

importance of weight loss approach for obese OSAHS subjects who received various therapies for OSAHS symptoms ${ }^{23)}$.

Obese OSAHS subjects showed more abnormal eating habits with regard to many component of dietary behavior compared with those of OSAHS subjects of normal weight ${ }^{24)}$. Weight loss approaches for obese workers with OSAHS are expected to improve worker's health condition as well as to prevent occupational accidents and to improve work productivity. There is also a possibility that increased workplace stress from job strain affects workers eating behaviors causing them to eat a lot and thus contributes to obesity among male Japanese workers $\left.{ }^{25}, 26\right)$. To more effectively promote weight loss among obese workers with OSAHS, it might be necessary to add stress management in the workplace to weight loss approach.

Our study has several limitations. First, there was no control group, and CPAP itself may also be a cause of weight loss in these subjects ${ }^{11,13)}$. However, the effect of CPAP may be small, because the average weight of subjects has increased by $1.8 \mathrm{~kg}$ over the prior year. Second, we did not examine sleep conditions using objective parameters such as oxygen desaturation index and apnea-hypopnea index, and did not select blood pressure, lipid levels, insulin resistance and waist circumstance as outcome measures of this study. The reason was that we put the feasibility of the weight loss program in a clinical setting before the full examination for evaluation of the program. In addition, we did not examine parameters of work performance. It is unclear whether such factors changed during the weight loss program and CPAP use and whether weight loss in this study was affected by such factors. Third, our followup period was short, 4 months. The long term effects of the present program remain unknown. Fourth, the number of our study participants was limited, and they were volunteers. This might have influenced the results, because of the possibility that there was a stronger motivation to ward weight loss among participants than there was among non participants. Further studies are warranted to determine the effect of the weight loss program for a general population of obese OSAHS subjects including those with weak motivation, and to clarify the influence job type on the efficacy of the program in grater number of participants.

In conclusion, we found that a weight loss program using individual counseling and behavioral approach was useful for promoting weight reduction among male obese OSAHS subjects receiving CPAP in a clinical setting. Furthermore, increased weight reduction in obese subjects with shorter CPAP duration compared to that in those with a longer duration of CPAP provides evidence supporting early referral to a weight loss program. We assume that effects of the weight loss approach indicate the importance of emphasizing weight loss approach with regularity in the workplaces of industrial health to worker's health condition and to prevent occupational accidents and decrease of work productivity. 


\section{Acknowledgements}

We are grateful to all the volunteers who participated in this study. This work was supported by Dokkyo Medical University, Young Investigator Award.

\section{References}

1) Lang A, Froelicher ES (2006) Management of overweight and obesity in adults: behavioral intervention for long-term weight loss and maintenance. Eur J Cardiovasc Nurs 5, 102-14.

2) Young T, Blustein J, Finn L, Palta M (1997) Sleepdisordered breathing and motor vehicle accidents in a population-based sample of employed adults. Sleep 20, 608-13.

3) Lindberg E, Carter N, Gislason T, Janson C (2001) Role of snoring and daytime sleepiness in occupational accidents. Am J Respir Crit Care Med 164, 2031-5.

4) Sivertsen B, Overland S, Glozier N, Bjorvatn B, Maeland JG, Mykletun A (2008) The effect of OSAS on sick leave and work disability. Eur Respir J 32, 1497-503.

5) Mulgrew AT, Ryan CF, Fleetham JA, Cheema R, Fox N, Koehoorn M, Fitzgerald JM, Marra C, Ayas NT (2007) The impact of obstructive sleep apnea and daytime sleepiness on work limitation. Sleep Med 9, 42-53.

6) Noseda A, Kempenaers C, Kerkhofs M, Houben JJ, Linkowski P (1996) Sleep apnea after 1 year domiciliary nasal-continuous positive airway pressure and attempted weight reduction. Potential for weaning from continuous positive airway pressure. Chest 109, 138-43.

7) Kajaste S, Brander PE, Telakivi T, Partinen M, Mustajoki P (2004) A cognitive-behavioral weight reduction program in the treatment of obstructive sleep apnea syndrome with or without initial nasal CPAP: a randomized study. Sleep Med 5, 125-31.

8) Tuomilehto HP, Seppa JM, Partinen MM, Peltonen, M, Gylling H, Tuomilehto JO, Vanninen E J, Kokkarinen J, Sahlman JK, Martikainen T, Soini EJ, Randell J, Tukiainen H, Uusitupa M (2009) Lifestyle intervention with weight reduction: first-line treatment in mild obstructive sleep apnea. Am J Respir Crit Care Med 179, 320-7.

9) McTigue KM, Harris R, Hemphill B, Lux L, Sutton S, Bunton AJ, Lohr KN (2003) Screening and interventions for obesity in adults: summary of the evidence for the U.S. Preventive Services Task Force. Ann Intern Med 139, 933-49.

10) Veasey SC, Guilleminault C, Strohl KP, Sanders MH, Ballard RD, Magalang UJ (2006) Medical therapy for obstructive sleep apnea: a review by the Medical Therapy for Obstructive Sleep Apnea Task Force of the Standards of Practice Committee of the American Academy of Sleep Medicine. Sleep 29, 1036-44.

11) Loube DI, Loube AA, Erman MK (1997) Continuous positive airway pressure treatment results in weight less in obese and overweight patients with obstructive sleep apnea. J Am Diet Assoc 97, 896-7.

12) Redenius R, Murphy C, O’Neill E, Al-Hamwi M, Zallek SN (2008) Does CPAP lead to change in BMI? J Clin Sleep Med 4, 205-9.

13) Kato-Nishimura K, Taniike M, Tachibana N (2008) Body weight reduction by CPAP treatment in a bedridden patient. Sleep Med 9, 207-8.

14) American Academy of Sleep Medicine (2005) Classification of Sleep Disorders, Diagnostic and coding manual. American Academy of Sleep Medicine, 2nd Ed., Westchester, Illinois.

15) Adachi Y, Kunitsuka K, Yamaguchi Y (2006) Self genryou manual. Niheisya Corp, Osaka (in Japanese).

16) Sano N, Sano S, Kataoka M, Chiba S, Yagi T, Okushi T, Endo M, Wada K, Yaguchi Y, Kotajima F, Mochizuki T, Kinoshita Y, Sugisaki M (2004) Objective evaluation of continuous positive airway pressure (CPAP). Compliance in obstructive sleep apnea syndrome (OSAS). Jikei Journal of Chest Diseases 16, 43-8 (in Japanese).

17) Buysse DJ, Reynolds CF 3rd, Monk TH, Berman SR, Kupfer DJ (1989) The Pittsburgh Sleep Quality Index: a new instrument for psychiatric practice and research. Psychiatry Res 28, 193-213.

18) Doi Y, Minowa M, Uchiyama M, Okawa K (1998) Pittsburgh Sleep Quality Index Japanese Version. Jpn J Psychiatr Treat 13, 755-63 (in Japanese).

19) Johns MW (1991) A new method for measuring daytime sleepiness: the Epworth sleepiness scale. Sleep 14, 540-5.

20) Takegami M, Sokejima S, Yamazaki S, Nakayama T, Fukuhara S (2005) An estimation of the prevalence of excessive daytime sleepiness based on age and sex distribution of epworth sleepiness scale scores: a population based survey. Nippon Koshu Eisei Zasshi 52, 137-45 (in Japanese).

21) Prochaska JO, DiClemente CC, Norcross JC (1992) In search of how people change. Applications to addictive behaviors. Am Psychol 47, 1102-14.

22) Labib M (2003) Acp Best Practice No 168. The investigation and management of obesity. J Clin Pathol 56, 17-25.

23) Suimin Kokyuu Syougai Kenyuukai (2005) Guideline for diagnosis \& treatment of adult SAS, Suimin Kokyuu Syougai Kenyuukai, Tokyo (in Japanese).

24) Fukushima S, Hisanaga A, Maeda H, Ohnishi K, Hosoi Y (2003) Analysis of the questionnaires of dietary behavior and the nutritional survey of food in the patients with sleep apnea syndrome. Nihon Syokugyo Saigai Igakkai Zasshi 51, 353-7 (in Japanese).

25) Nishitani N, Sakakibara H (2006) Relationship of obesity to job stress and eating behavior in male Japanese workers. Int J Obes 30, 528-33.

26) Nishitani N, Sakakibara H, Akiyama I (2009) Eating behavior related to obesity and job stress in male Japanese workers. Nutrition 25, 45-50. 